\title{
SMEFT Constraints on New Physics beyond the Standard Model
}

\author{
John Ellis ${ }^{1,2,3}$ \\ ${ }^{1}$ Department of Physics, King's College London, London WC2R 2LS, United Kingdom \\ ${ }^{2}$ National Institute of Chemical Physics E Biophysics, Rävala 10, 10143 Tallinn, Estonia \\ ${ }^{3}$ Theoretical Physics Department, CERN, CH-1211 Geneva 23, Switzerland
}

\begin{abstract}
The Fermi effective theory of the weak interaction helped identify the structure of the electroweak sector of the Standard Model, and the chiral effective Lagrangian pointed towards QCD as the theory of the strong interactions. The Standard Model Effective Field Theory (SMEFT) is a systematic and model-independent framework for characterizing experimental deviations from the predictions of the Standard Model and pointing towards the structures of its possible extensions that is complementary to direct searches for new physics beyond the Standard Model. This talk summarizes results from the first global fit to data from LHC Run 2 and earlier experiments including dimension-6 SMEFT operators, and gives examples how it can be used to constrain scenarios for new physics beyond the Standard Model. In addition, some windows for probing dimension-8 SMEFT operators are also mentioned.
\end{abstract}

Keywords: standard model, Higgs, effective field theory, collider experiments, new physics

KCL-PH-TH/2021-035, CERN-TH-2021-083

DOI: 10.31526/ACP.BSM-2021.16

\section{INTRODUCTION}

Since its formulation 54 years ago, many experiments have verified predictions of the Standard Model (SM). The $\mathrm{SU}(3) \times \mathrm{SU}(2) \times \mathrm{U}(1)$ structure of its gauge interactions has been well established, as have the representations and quantum numbers of the matter fermions. Predictions based on these properties were tested below the $0.1 \%$ level by experiments at LEP and other accelerators before the LHC was put into operation [1]. However, there was no direct evidence for the existence of the Higgs field, its effective potential and its Yukawa interactions with matter fermions before LHC operations started. Following the discovery of the Higgs boson in 2012 [2], these aspects of the SM are now also being studied, and measured with increasing precision.

So far, all LHC measurements of cross sections for the production of SM particles are in agreement with theoretical predictions, within the uncertainties in both theory and experiment. These include the production of $W$ and $Z$ bosons alone or with multiple QCD jets, diboson pairs, triple-boson combinations, $t \bar{t}$ pairs (also with a $\gamma, W$ or $Z$ boson), and single $t$ production alone or accompanied by a $\gamma, W$ or $Z$ boson [3].

The SM has also been successful with its predictions for the production of the Higgs boson by gluon collisions, vector-boson fusion, in association with a $W$ or $Z$, or together with a $t \bar{t}$ pairs. The SM predictions for many Higgs decays have also been successful. These have enabled the couplings of the Higgs boson to many SM particles, including the $\mu, \tau, b, W, Z$ and $t$ to be measured. So far, the measurements are consistent with being proportional to the corresponding particle masses, as expected in the SM [4]. As we wrote a few years back "it walks and quacks like a Higgs boson" [5].

However, everything about the Higgs boson is puzzling. Consider the terms in the SM Lagrangian that involve the Higgs field:

$$
\mathcal{L}=y H \bar{\psi} \psi+\mu^{2}|H|^{2} \lambda|H|^{4}-V_{0}+\ldots
$$

The pattern of Yukawa couplings $y$ is completely mysterious, and intimately related to the flavour problems of the SM: what is the origin of the hierarchies of fermion masses, the undeciphered matrix of quark mixing and the origin of $\mathrm{CP}$ violation in the quark sector? The sign and magnitude of the coefficient $\mu^{2}$ of the second term in (1) is equally baffling: in particular, how and why is $|\mu|$ many orders of magnitude than the Planck scale? This is often termed the naturalness or hierarchy problem. The magnitude of the quartic coupling $\lambda$ is also baffling. According to SM calculations, $\lambda$ is so small that it would get renormalized to negative values at high scales [6] by the Higgs coupling to the top quark, in which case the current electroweak vacuum would in principle be unstable, and most of the Universe would not have reached it during the evolution of the Universe [7]. The $V_{0}$ term in (1) is equivalent to Einstein's cosmological constant or today's dark energy. This receives contributions from spontaneous symmetry breaking and non-perturbative QCD effects that are many orders of magnitude larger than the measured value. Here the mystery is not why $V_{0} \neq 0$, but rather why it is so small, over 120 orders of magnitude smaller than the "natural" gravitational scale.

How should we search for new physics beyond the Standard Model? This where the ... in (1) come in. There could be additional interactions beyond those in the SM, of dimension 6 or more, which could be generated by the exchanges of massive particles as yet undetected. These might involve the Higgs field alone, or in combination with other SM fields, or only other fields. They should all be analyzed together in order for the indirect search for new physics beyond the SM via higher-dimensional interactions to be as powerful and model-independent as possible. 
In looking for new physics, we may keep in mind the advice of the Chinese strategist Sun Tzu [8]:

"...the direct method may be used...but indirect methods will be needed in order to secure victory.... The direct and the indirect lead on to each other in turn. It is like moving in a circle... Who can exhaust the possibilities of their combination?"

This is the philosophy behind the use of the Standard Model Effective Field Theory described in the following.

\section{THE STANDARD MODEL EFFECTIVE FIELD THEORY (SMEFT)}

Effective Field Theories (EFTs) have a long and glorious history in particle physics.

Back in the 1930s, the interaction between radiation and electrons was described by an embryonic form of QED, described by a dimension-4 Lagrangian term. However, the weak interactions fell outside this framework, and Fermi proposed an effective four-fermion interaction to describe them [9], described by some combination of operators of dimension 6 . The natural expectation was that this interaction was mediated by the exchange of massive bosons. However, for many years the Lorentz structure of the four-fermion interaction was unclear: scalar/pseudoscalar, vector/axial vector or tensor, and hence also the spins of the exchanged bosons. Finally, it was established in the 1950s that the interaction was of $V-A$ form [10], so that the intermediate boson(s) should have spin one. This observation pointed towards gauge theory, but for a decade it was unknown how to give masses to the bosons. This problem was solved in the 1960s by the discovery of the Englert-Brout-Higgs mechanism [11] and its application in the Standard Model.

In parallel during the 1930s, Yukawa proposed a theory of the strong nucleon-nucleon force according to which it was mediated by mesons, of which the lightest was the pion that was discovered in the 1940s. It was later discovered that the interactions of the pions involved field derivatives, e.g., the dominant contribution to $\pi \pi$ scattering is an interaction of the form $\partial \pi \partial \pi \pi \pi$. This structure is a manifestation of an underlying chiral dynamics, which is an important clue to the nature of the structure of the underlying forces between quarks. In particular, it implied that the exchanged gluons should also be vector (rather than scalar) bosons, pointing the way towards QCD.

Will the EFT approach now provide us hints how to progress beyond the Standard Model? Many extensions of the Standard Model postulate heavy particles whose exchanges could generate effective interactions of dimensions higher than 4 whose structure may give valuable information about the structure about extensions of the Standard Model. A first example is provided by the light neutrino masses, which can be described by an effective interaction of dimension 5 [12] likely to be generated by the exchanges of massive fermions. The experimental fact that neutrino flavours mix in a very different way from quarks is presumably a consequence of this underlying structure, and may give us a hint about new physics far above the electroweak scale.

The Standard Model Effective Field Theory (SMEFT) $[13,14]$ provides a very powerful framework for analyzing in a global way many LHC and other measurements. One assumes that the Standard Model assignments of the quantum numbers of the Standard Model particles are correct, which determine the structure of the dimension $\leq 4$ terms in the Lagrangian, but accepts that it is incomplete. One allows for additional higher-order interactions between the known particles that respect the symmetries of the Standard Model, and constrains their strengths via a joint analysis of Higgs data together with electroweak precision measurements, diboson [15] and top data [16]. This analysis can then be used to look in a general and model-independent way for possible physics beyond the Standard Model.

There are 2499 possible operators $\mathcal{O}_{i}$ in the dimension-6 part of the SMEFT Lagrangian:

$$
\mathcal{L}_{\mathrm{SMEFT}}=\mathcal{L}_{\mathrm{SM}}+\sum_{i=1}^{2499} \frac{C_{i}}{\Lambda^{2}} \mathcal{O}_{i},
$$

where $\Lambda$ is the new physics scale, which is assumed to be much larger than the masses of the Standard Model particles and the kinematic scales of the processes studied, and the $C_{i}$ are operator coefficients whose magnitudes depend, e.g., whether the underlying massive particles are weakly- or strongly-interacting. It is clearly impractical to include 2499 parameters in a global fit, so one generally makes simplifying assumptions about the flavour structure of the operators involving fermions. In the following we consider two possibilities [16]: a flavour-universal scenario in which their coefficients have an SU(3) ${ }^{5}$ symmetry, and a topspecific scenario in which the symmetry is reduced to $\mathrm{SU}(2)^{2} \times \mathrm{SU}(3)^{3}$. When making our global fit, we treat $G_{F}, M_{Z}$ and the fine structure constant $\alpha$ as input parameters, and work to linear order in the operator coefficients, i.e, we consider only the interferences of the $\mathcal{O}_{i}$ with the Standard Model interactions. Thus, we work to order $1 / \Lambda^{2}$ and neglect consistently dimension- 8 interactions that would contribute at $\mathcal{O}\left(1 / \Lambda^{4}\right)$.

The Table below lists all the relevant dimension-6 SMEFT operators, including those containing two and four fermions, which are important when considering top observables. The grey cells contain operators that violate $\mathrm{SU}(3)^{5}$ universality. If we impose universality, the following 20 operators affect primarily electroweak precision observables, bosonic observables and Yukawa couplings, respectively:

$$
\begin{aligned}
\text { EWPO: } & \mathcal{O}_{H W B}, \mathcal{O}_{H D}, \mathcal{O}_{l l}, \mathcal{O}_{H l}^{(3)}, \mathcal{O}_{H l}^{(1)}, \mathcal{O}_{H e}, \mathcal{O}_{H q}^{(3)}, \mathcal{O}_{H q}^{(1)}, \mathcal{O}_{H d}, \mathcal{O}_{H u}, \\
\text { Bosonic: } & \mathcal{O}_{H \square}, \mathcal{O}_{H G}, \mathcal{O}_{H W}, \mathcal{O}_{H B}, \mathcal{O}_{W}, \mathcal{O}_{G}, \\
\text { Yukawa: } & \mathcal{O}_{\tau H}, \mathcal{O}_{\mu H}, \mathcal{O}_{b H}, \mathcal{O}_{t H} .
\end{aligned}
$$




\begin{tabular}{|c|c|c|c|c|c|}
\hline \multicolumn{2}{|r|}{$X^{3}$} & \multicolumn{2}{|r|}{$H^{6}$ and $H^{4} D^{2}$} & \multicolumn{2}{|r|}{$\psi^{2} H^{3}$} \\
\hline $\mathcal{O}_{G}$ & $f^{A B C} G_{\mu}^{A v} G_{v}^{B \rho} G_{\rho}^{C \mu}$ & $\mathcal{O}_{H}$ & $\left(H^{\dagger} H\right)^{3}$ & $\mathcal{O}_{e H}$ & $\left(H^{\dagger} H\right)\left(\bar{l}_{p} e_{r} H\right)$ \\
\hline $\mathcal{O}_{\widetilde{G}}$ & $f^{A B C} \widetilde{G}_{\mu}^{A v} G_{v}^{B \rho} G_{\rho}^{C \mu}$ & $\mathcal{O}_{H \square}$ & $\left(H^{\dagger} H\right) \square\left(H^{\dagger} H\right)$ & $\mathcal{O}_{u H}$ & $\left(H^{\dagger} H\right)\left(\bar{q}_{p} u_{r} \widetilde{H}\right)$ \\
\hline $\mathcal{O}_{W}$ & $\varepsilon^{I J K} W_{\mu}^{I \nu} W_{v}^{J \rho} W_{\rho}^{K \mu}$ & $\mathcal{O}_{H D}$ & $\left(H^{\dagger} D^{\mu} H\right)^{\star}\left(H^{\dagger} D_{\mu} H\right)$ & $\mathcal{O}_{d H}$ & $\left(H^{\dagger} H\right)\left(\bar{q}_{p} d_{r} H\right)$ \\
\hline $\mathcal{O}_{\tilde{W}}$ & $\varepsilon^{I J K} \widetilde{W}_{\mu}^{I v} W_{v}^{J \rho} W_{\rho}^{K \mu}$ & & & & \\
\hline \multicolumn{2}{|r|}{$\mathrm{X}^{2} H^{2}$} & \multicolumn{2}{|r|}{$\psi^{2} X H$} & \multicolumn{2}{|r|}{$\psi^{2} H^{2} D$} \\
\hline $\mathcal{O}_{H G}$ & $H^{\dagger} H G_{\mu \nu}^{A} G^{A \mu \nu}$ & $\mathcal{O}_{e W}$ & $\left(\bar{l}_{p} \sigma^{\mu v} e_{r}\right) \tau^{I} H W_{\mu v}^{I}$ & $\mathcal{O}_{H l}^{(1)}$ & $\left(H^{\dagger} i \stackrel{\leftrightarrow}{D_{\mu}} H\right)\left(\bar{l}_{p} \gamma^{\mu} l_{r}\right)$ \\
\hline $\mathcal{O}_{H \tilde{G}}$ & $H^{\dagger} H \widetilde{G}_{\mu \nu}^{A} G^{A \mu \nu}$ & $\mathcal{O}_{e B}$ & $\left(\bar{l}_{p} \sigma^{\mu v} e_{r}\right) H B_{\mu v}$ & $\mathcal{O}_{H l}^{(3)}$ & $\left(H^{+} i \stackrel{\leftrightarrow}{\mu}_{\stackrel{\leftrightarrow}{I}}^{\leftrightarrow} H\right)\left(\bar{l}_{p} \tau^{I} \gamma^{\mu} l_{r}\right)$ \\
\hline $\mathcal{O}_{H W}$ & $H^{\dagger} H W_{\mu \nu}^{I} W^{I \mu \nu}$ & $\mathcal{O}_{u G}$ & $\left(\bar{q}_{p} \sigma^{\mu \nu} T^{A} u_{r}\right) \widetilde{H} G_{\mu \nu}^{A}$ & $\mathcal{O}_{H e}$ & $\left(H^{+} i \stackrel{\leftrightarrow}{D_{\mu}} H\right)\left(\bar{e}_{p} \gamma^{\mu} e_{r}\right)$ \\
\hline $\mathcal{O}_{H \widetilde{W}}$ & $H^{\dagger} H \widetilde{W}_{\mu \nu}^{I} W^{I \mu \nu}$ & $\mathcal{O}_{u W}$ & $\left(\bar{q}_{p} \sigma^{\mu v} u_{r}\right) \tau^{I} \widetilde{H} W_{\mu \nu}^{I}$ & $\mathcal{O}_{\mathrm{Hq}}^{(1)}$ & $\left(H_{\leftrightarrow}^{+} i_{\mu} H\right)\left(\bar{q}_{p} \gamma^{\mu} q_{r}\right)$ \\
\hline $\mathcal{O}_{H B}$ & $H^{\dagger} H B_{\mu \nu} B^{\mu \nu}$ & $\mathcal{O}_{u B}$ & $\left(\bar{q}_{p} \sigma^{\mu v} u_{r}\right) \widetilde{H} B_{\mu v}$ & $\mathcal{O}_{H q}^{(3)}$ & $\left(H^{\dagger} i \stackrel{\leftrightarrow}{\mu}_{\leftrightarrow}^{I} H\right)\left(\bar{q}_{p} \tau^{I} \gamma^{\mu} q_{r}\right)$ \\
\hline $\mathcal{O}_{H \tilde{B}}$ & $H^{+} H \widetilde{B}_{\mu \nu} B^{\mu v}$ & $\mathcal{O}_{d G}$ & $\left(\bar{q}_{p} \sigma^{\mu v} T^{A} d_{r}\right) H G_{\mu v}^{A}$ & $\mathcal{O}_{H u}$ & $\left(H^{\dagger} i D_{\mu} H\right)\left(\bar{u}_{p} \gamma^{\mu} u_{r}\right)$ \\
\hline $\mathcal{O}_{H W B}$ & $H^{\dagger} \tau^{I} H W_{\mu \nu}^{I} B^{\mu \nu}$ & $\mathcal{O}_{d W}$ & $\left(\bar{q}_{p} \sigma^{\mu v} d_{r}\right) \tau^{I} H W_{\mu \nu}^{I}$ & $\mathcal{O}_{H d}$ & 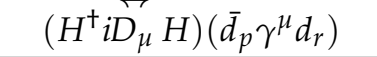 \\
\hline $\mathcal{O}_{H \tilde{W} B}$ & $H^{\dagger} \tau^{I} H \widetilde{W}_{\mu v}^{I} B^{\mu \nu}$ & $\mathcal{O}_{d B}$ & $\left(\bar{q}_{p} \sigma^{\mu v} d_{r}\right) H B_{\mu v}$ & $\mathcal{O}_{\text {Hud }}$ & $i\left(\widetilde{H}^{\dagger} D_{\mu} H\right)\left(\bar{u}_{p} \gamma^{\mu} d_{r}\right)$ \\
\hline \multicolumn{2}{|r|}{$(\bar{L} L)(\bar{L} L)$} & \multicolumn{2}{|r|}{$(\bar{R} R)(\bar{R} R)$} & \multicolumn{2}{|r|}{$(\bar{L} L)(\bar{R} R)$} \\
\hline $\mathcal{O}_{l l}$ & $\left(\bar{l}_{p} \gamma_{\mu} l_{r}\right)\left(\bar{l}_{s} \gamma^{\mu} l_{t}\right)$ & $\mathcal{O}_{e e}$ & $\left(\bar{e}_{p} \gamma_{\mu} e_{r}\right)\left(\bar{e}_{s} \gamma^{\mu} e_{t}\right)$ & $\mathcal{O}_{l e}$ & $\left(\bar{l}_{p} \gamma_{\mu} l_{r}\right)\left(\bar{e}_{s} \gamma^{\mu} e_{t}\right)$ \\
\hline $\mathcal{O}_{q q}^{(1)}$ & $\left(\bar{q}_{p} \gamma_{\mu} q_{r}\right)\left(\bar{q}_{s} \gamma^{\mu} q_{t}\right)$ & $\mathcal{O}_{u u}$ & $\left(\bar{u}_{p} \gamma_{\mu} u_{r}\right)\left(\bar{u}_{s} \gamma^{\mu} u_{t}\right)$ & $\mathcal{O}_{l u}$ & $\left(\bar{l}_{p} \gamma_{\mu} l_{r}\right)\left(\bar{u}_{s} \gamma^{\mu} u_{t}\right)$ \\
\hline $\mathcal{O}_{q q}^{(3)}$ & $\left(\bar{q}_{p} \gamma_{\mu} \tau^{I} q_{r}\right)\left(\bar{q}_{s} \gamma^{\mu} \tau^{I} q_{t}\right)$ & $\mathcal{O}_{d d}$ & $\left(\bar{d}_{p} \gamma_{\mu} d_{r}\right)\left(\bar{d}_{s} \gamma^{\mu} d_{t}\right)$ & $\mathcal{O}_{l d}$ & $\left(\overline{\bar{l}}_{p} \gamma_{\mu} l_{r}\right)\left(\bar{d}_{s} \gamma^{\mu} d_{t}\right)$ \\
\hline $\mathcal{O}_{l q}^{(1)}$ & $\left(\bar{l}_{p} \gamma_{\mu} l_{r}\right)\left(\bar{q}_{s} \gamma^{\mu} q_{t}\right)$ & $\mathcal{O}_{e u}$ & $\left(\bar{e}_{p} \gamma_{\mu} e_{r}\right)\left(\bar{u}_{s} \gamma^{\mu} u_{t}\right)$ & $\mathcal{O}_{q e}$ & $\left(\bar{q}_{p} \gamma_{\mu} q_{r}\right)\left(\bar{e}_{s} \gamma^{\mu} e_{t}\right)$ \\
\hline \multirow[t]{3}{*}{$\mathcal{O}_{l q}^{(3)}$} & $\left(\bar{l}_{p} \gamma_{\mu} \tau^{I} l_{r}\right)\left(\bar{q}_{s} \gamma^{\mu} \tau^{I} q_{t}\right)$ & $\mathcal{O}_{e d}$ & $\left(\bar{e}_{p} \gamma_{\mu} e_{r}\right)\left(\bar{d}_{s} \gamma^{\mu} d_{t}\right)$ & $\mathcal{O}_{q u}^{(1)}$ & $\left(\bar{q}_{p} \gamma_{\mu} q_{r}\right)\left(\bar{u}_{s} \gamma^{\mu} u_{t}\right)$ \\
\hline & & $\mathcal{O}_{u d}^{(1)}$ & $\begin{array}{c}\left(\bar{u}_{p} \gamma_{\mu} u_{r}\right)\left(\bar{d}_{s} \gamma^{\mu} d_{t}\right) \\
u^{A} u_{1}\end{array}$ & $\mathcal{O}_{q u}^{(8)}$ & $\left(\bar{q}_{p} \gamma_{\mu} T^{A} q_{r}\right)\left(\bar{u}_{s} \gamma^{\mu} T^{A} u_{t}\right)$ \\
\hline & & $\mathcal{O}_{u d}^{(0)}$ & $\left(\bar{u}_{p} \gamma_{\mu} T^{A} u_{r}\right)\left(d_{s} \gamma^{\mu} T^{A} d_{t}\right)$ & $\begin{array}{l}\mathcal{O}_{q d}^{(1)} \\
\mathcal{O}_{q d}^{(8)}\end{array}$ & $\begin{array}{c}\left(\bar{q}_{p} \gamma_{\mu} q_{r}\right)\left(d_{s} \gamma^{\mu} d_{t}\right) \\
\left(\bar{q}_{p} \gamma_{\mu} T^{A} q_{r}\right)\left(\bar{d}_{s} \gamma^{\mu} T^{A} d_{t}\right)\end{array}$ \\
\hline \multicolumn{2}{|c|}{$(\bar{L} R)(\bar{R} L)$ and $(\bar{L} R)(\bar{L} R)$} & \multicolumn{4}{|c|}{$B$-violating } \\
\hline $\mathcal{O}_{\text {ledq }}$ & $\left(\bar{l}_{p}^{j} e_{r}\right)\left(\bar{d}_{s} q_{t}^{j}\right)$ & $\mathcal{O}_{d u q}$ & $\varepsilon^{\alpha \beta \gamma} \varepsilon_{j k}[(\alpha$ & $\left.{ }^{T} C u_{r}^{\beta}\right]$ & $\left.\left(q_{s}^{\gamma j}\right)^{T} C l_{t}^{k}\right]$ \\
\hline $\mathcal{O}_{q u q d}^{(1)}$ & $\left(\bar{q}_{p}^{j} u_{r}\right) \varepsilon_{j k}\left(\bar{q}_{s}^{k} d_{t}\right)$ & $\mathcal{O}_{q q u}$ & $\varepsilon^{\alpha \beta \gamma} \varepsilon_{j k}[(q$ & ${ }^{T} C q_{r}^{\beta k}$ & {$\left[\left(u_{s}^{\gamma}\right)^{T} C e_{t}\right]$} \\
\hline $\mathcal{O}_{q u q d}^{(8)}$ & $\left(\bar{q}_{p}^{j} T^{A} u_{r}\right) \varepsilon_{j k}\left(\bar{q}_{s}^{k} T^{A} d_{t}\right)$ & $\mathcal{O}_{q q q}$ & $\varepsilon^{\alpha \beta \gamma} \varepsilon_{j n} \varepsilon_{k m}[($ & ${ }^{T} C q_{r}^{\beta \bar{k}}$ & {$\left[\left(q_{s}^{\gamma m}\right)^{T} C l_{t}^{n}\right]$} \\
\hline $\mathcal{O}_{\text {lequ }}^{(1)}$ & $\left(\bar{l}_{p}^{j} e_{r}\right) \varepsilon_{j k}\left(\bar{q}_{s}^{k} u_{t}\right)$ & $\mathcal{O}_{\text {duи }}$ & $\varepsilon^{\alpha \beta \gamma}\left[\left(d_{1}^{a}\right.\right.$ & $\left.C u_{r}^{\beta}\right][$ & $\left.\left.u_{s}^{\gamma}\right)^{T} C e_{t}\right]$ \\
\hline $\mathcal{O}_{\text {lequ }}^{(3)}$ & $\left(\bar{l}_{p}^{j} \sigma_{\mu v} e_{r}\right) \varepsilon_{j k}\left(\bar{q}_{s}^{k} \sigma^{\mu v} u_{t}\right)$ & & & & \\
\hline
\end{tabular}

Dimension-6 operators considered in [16]. The operators in cells shaded grey break flavour SU(3) ${ }^{5}$ explicitly.

Another 14 two- and four-fermion operators appear when we break the flavour universality down to $\mathrm{SU}(2)^{2} \times \mathrm{SU}(3)^{3}$ in the topspecific scenario:

$$
\begin{array}{ll}
\text { Top 2F: } & \mathcal{O}_{H Q}^{(3)}, \mathcal{O}_{H Q}^{(1)}, \mathcal{O}_{H t}, \mathcal{O}_{t G}, \mathcal{O}_{t W}, \mathcal{O}_{t B}, \\
\text { Top 4F: } & \mathcal{O}_{Q q}^{3,1}, \mathcal{O}_{Q q}^{3,8}, \mathcal{O}_{Q q}^{1,8}, \mathcal{O}_{Q u}^{8}, \mathcal{O}_{Q d}^{8}, \mathcal{O}_{t Q}^{8}, \mathcal{O}_{t u}^{8}, \mathcal{O}_{t d}^{8} .
\end{array}
$$

Results from global fits under these two flavour assumptions are discussed in the following Section.

\section{GLOBAL SMEFT FITS TO TO ELECTROWEAK, DIBOSON, HIGGS AND TOP DATA}

This Section presents some of the results from global fits to the dimension- 6 operators listed above, using precision electroweak data and $W^{+} W^{-}$data from LEP, as well as Higgs, diboson and top data from Runs 1 and 2 of the LHC: please see [16] for the complete results. Fig. 1 shows schematically how the different operators contribute to various sets of measurements. It emphasizes their interdependence, and hence the need for a global analysis taking all the data sets into consideration. Overall, we included 328 individual data points in our analysis, including separate bins characterizing kinematic variables in many differential distributions.

Fig. 2 shows the overall results from our flavour-universal $\mathrm{SU}(3)^{5}$-symmetric fit. The upper pair of panels shows the constraints we obtain on the operators when they are each switched on individually, whereas in the lower pair of panels all the 20 operators 


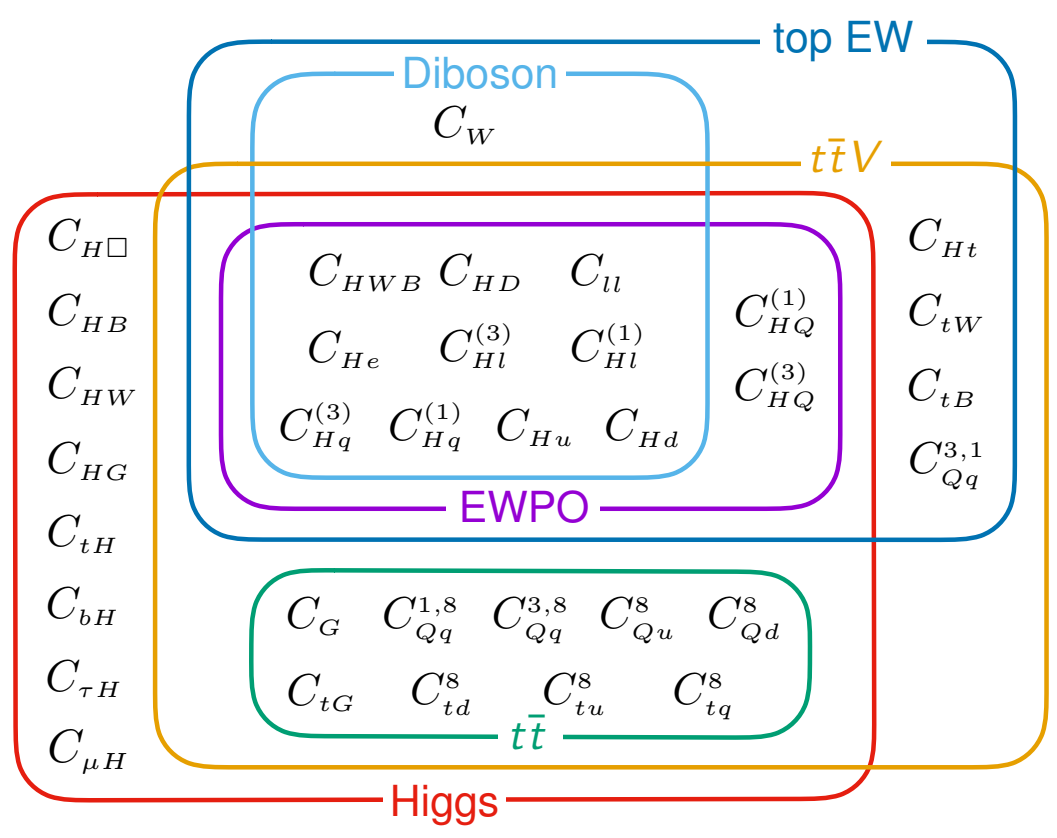

FIGURE 1: An illustration [16] how various classes of observables depend on the 34 dimension-6 operators $(3,4)$.

are switched on simultaneously, and the constraints on the operators are those obtained by marginalizing over the coefficients of all the other operators. The upper panel in each pairs shows the constraints on the numerical coefficients $C_{i}$ scaled for an operator scale $\Lambda=1 \mathrm{TeV}$, and the lower panel in each pair shows the constraints on $\Lambda$ obtained for various indicated values of the $C_{i}$. The colours in the histograms show the effects of including only Run-1 Higgs data, also Run-2 data, kinematic information denoted by STXS [17], and dropping data on $Z+$ dijet data.

In the case of the individual fit we see that the obtained constraints on $\Lambda$ are all in the multi-TeV range in the strong-coupling limit $C_{i}=(4 \pi)^{2}$, in which case whereas the linear treatment of the EFT approximation used here should be quite accurate. For $C_{i}=1$, the individual limits are still $\gtrsim 1 \mathrm{TeV}$, except for $C_{G}$, which is better constrained by a quadratic treatment of multi-jet data. On the other hand, several of the constraints on other operator scales get weaker than $1 \mathrm{TeV}$ in the weak-coupling case $C_{i}=0.01$. Also we see in the lower panels of Fig. 2 that all the constraints are significantly weaker in the marginalized fit, as could be expected in a fit with more parameters.

Fig. 3 shows corresponding results for the top-specific $\mathrm{SU}(2)^{2} \times \mathrm{SU}(3)^{3}$-symmetric fit with 34 operators. We see that the constraints in the electroweak, bosonic and Yukawa sectors are similar to those in the universal SU(3) ${ }^{5}$-symmetric fit. However, the constraints in the two- and four-fermion sectors are generally weaker. The constraints on $\Lambda$ are generally $\gtrsim 10 \mathrm{TeV}$ in the individual strong-coupling fit, but fall to $\gtrsim 300 \mathrm{GeV}$ in the marginalized fit with $C_{i}=1$. The linear EFT approximation is likely to break down in the top sector for the weak-coupling case $C_{i}=0.01$. $^{1}$

As seen in Fig. 1, many SMEFT operators contribute to the same data sectors, introducing correlations between the constraints on their coefficients. Moreover, many also contribute to multiple data sectors, introducing correlations between sectors. Fig. 4 displays the full $34 \times 34$ correlation matrix for the operator coefficients in the marginalized top-specific fit. We see substantial operator correlations within the EWPO and bosonic sectors, and also within the two- and four-fermion top sectors, as could be expected. However, correlations between operators in these sectors and in the Yukawa sector can also be significant, as reflected in the numerical values in off-diagonal squares in the matrix.

Fig. 5 illustrates an example of the interplay between the different constraints on a subset of operator coefficients, namely $\left\{C_{t H}, C_{t G}, C_{G}, C_{H G}\right\}$. The Higgs data provide only weak constraints (yellow shading) before the $t \bar{t} H$ measurements are included (green shading), and substantial further improvements are provided by including all the top data (pink shading) and four-fermion constraints (blue shading). Two points to note are the strong correlations between pairs of operators, and the fact that the Standard Model point is slightly outside the $95 \%$ confidence level region. As discussed in [16], this effect is largely driven by the measurement of the $t \bar{t}$ cross section close to threshold, which favours a non-zero value of $C_{G}$. However, multijet data impose a strong upper limit on $C_{G}$ when analyzed at quadratic order (not considered here), suggesting that an alternative explanation of the apparent $t \bar{t}$ threshold discrepancy may be needed.

Fig. 6 shows the results of a principal component analysis of the correlated constraints. The constraints on the scales $\Lambda$ of eigenvectors of the global likelihood function, normalizing the associated coefficient to unity, are shown in the left panel, in order of decreasing sensitivity. The relative constraining powers of different data sectors are shown in the right panel, and the compositions

\footnotetext{
${ }^{1}$ For results in the quadratic EFT approximation assuming that the precision electroweak data agree perfectly with the Standard Model and ignoring possible dimension-8 operator contributions, see [18].
} 

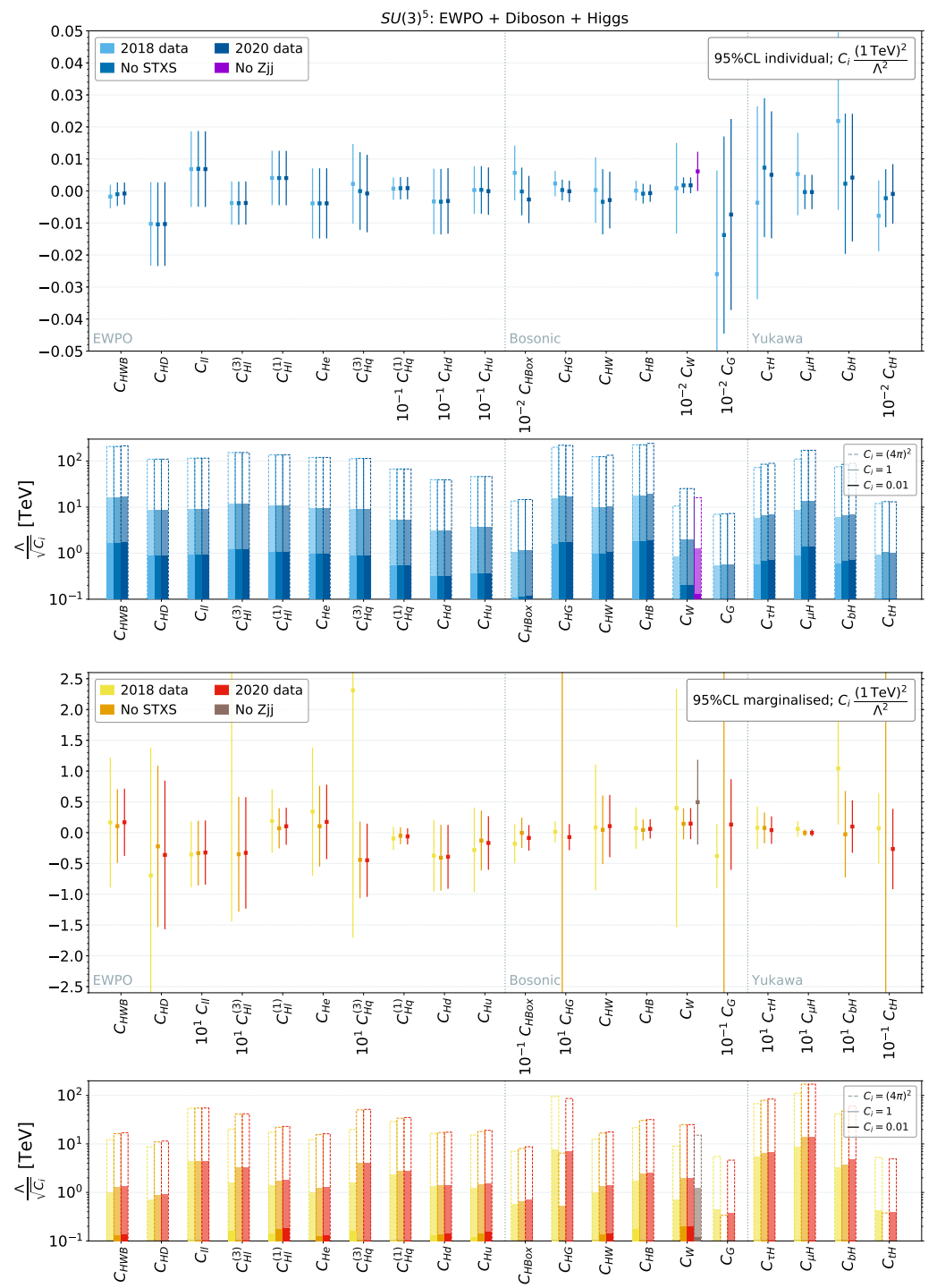

FIGURE 2: Constraints at the 95\% confidence level in the flavour-universal SU(3) ${ }^{5}$ scenario on individual and marginalized operator coefficients $C_{i}(1 \mathrm{TeV})^{2} / \Lambda^{2}$ (top and third panels) and the corresponding scales $\Lambda$ for the indicated values of the $C_{i}$ (second and bottom panels), from a combined linear fit to the Higgs, diboson and electroweak precision observables using the data sets indicated by colour coding [16].

of the eigenvectors are colour-coded in the central panel. We see that the least-constrained eigenvectors are only constrained by data on $t \bar{t}$ production, single top production, and $t \bar{t} V$ production. This reflects the fact that, as we already saw in Fig. 3 , the precision of top data currently lags behind that in the other data sectors.

\section{SINGLE-FIELD EXTENSIONS OF THE STANDARD MODEL}

As a first example of the constraints that our analysis places on specific scenarios for physics beyond the Standard Model, we considered in [16] the single-field extensions of the Standard Model catalogued in the Table below. As indicated, each of these models may be characterized by the mass of the additional field and a coupling constant. The tree-level contributions of each of these single-field extensions to dimension- 6 operator coefficients is given in [19]. These results can be combined with our global analysis to constrain the mass of the additional field (assuming that the corresponding coupling is unity) or the coupling (assuming that the corresponding mass is $1 \mathrm{TeV}$ ), as shown in Fig. 7. As noted, there is just a handful of instances where there is a deviation from the Standard Model that exceeds $1 \sigma$, none of which is significant. We find, in particular, that in all the possible extensions with a single spin-zero field the particle mass must exceed about $800 \mathrm{GeV}$, while the mass of any extra vector field must exceed about $1.5 \mathrm{TeV}$, if the corresponding coupling is unity. 

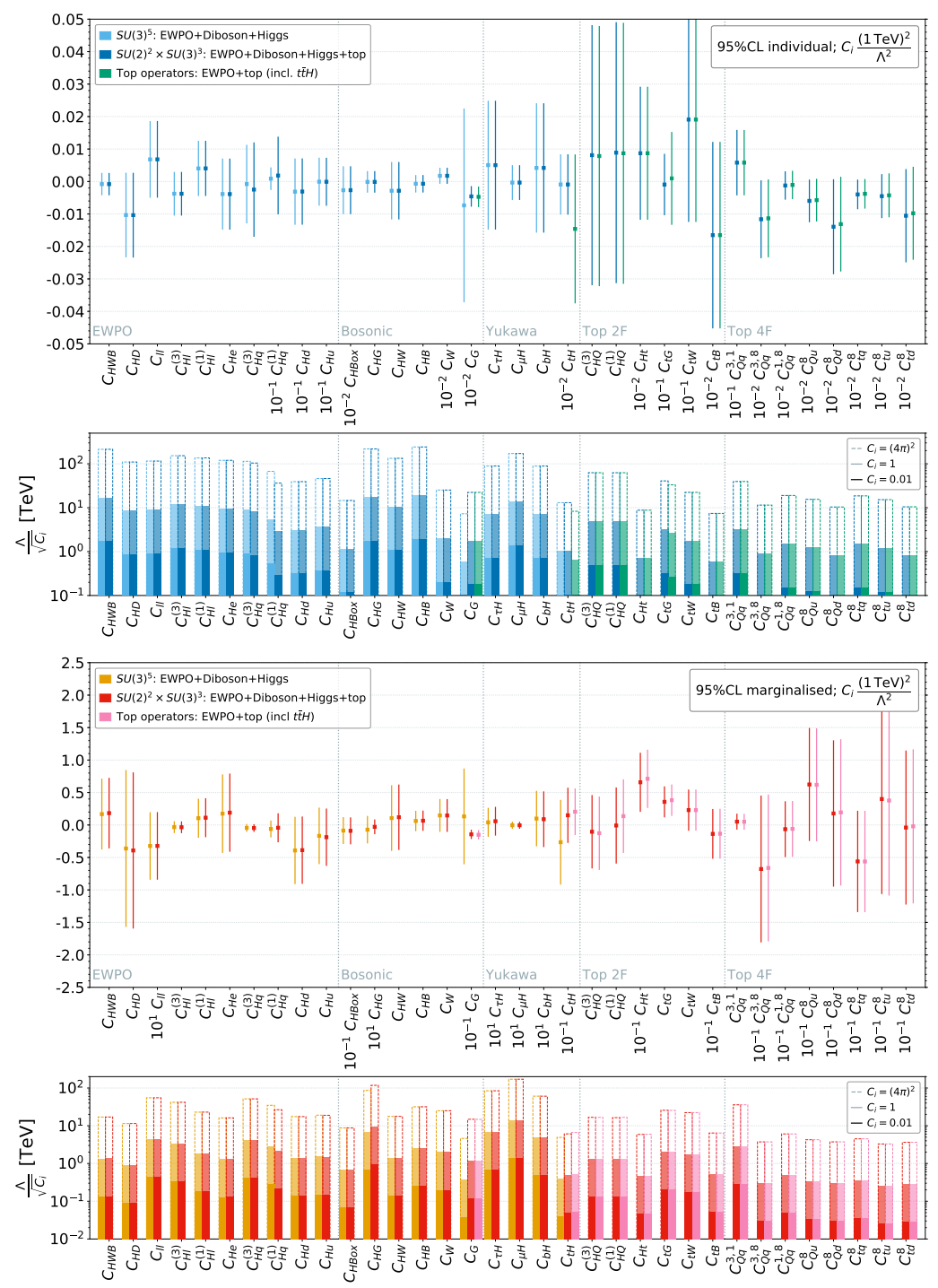

FIGURE 3: Constraints at the 95\% confidence level in the top-specific SU(2) ${ }^{2} \times S U(3)^{3}$ scenario on individual and marginalized operator coefficients $C_{i}(1 \mathrm{TeV})^{2} / \Lambda^{2}$ (top and third panels) and the corresponding scales $\Lambda$ for the indicated values of the $C_{i}$ (second and bottom panels), from a combined linear fit to the Higgs, diboson and electroweak precision observables using the data sets indicated by colour coding [16].

\section{SMEFT CONSTRAINTS ON LIGHT STOPS}

Another example of a search for new physics beyond the Standard Model using the SMEFT is provided by light stop squarks in supersymmetric models conserving $R$-parity [20]. In this case there is no tree-level contribution to any operator coefficients, but four coefficients receive one-loop contributions from stop squarks [21]:

$$
\begin{aligned}
C_{H G} & =\frac{g_{s}^{2}}{12} \frac{h_{t}^{2}}{(4 \pi)^{2}}\left[\left(1+\frac{1}{12} \frac{c_{2 \beta} g^{\prime 2}}{h_{t}^{2}}\right)-\frac{1}{2} \frac{X_{t}^{2}}{m_{\tilde{t}}^{2}}\right], \\
C_{H B} & =\frac{17 g^{\prime 2}}{144} \frac{h_{t}^{2}}{(4 \pi)^{2}}\left[\left(1+\frac{31}{102} \frac{c_{2 \beta} g^{\prime 2}}{h_{t}^{2}}\right)-\frac{38}{85} \frac{X_{t}^{2}}{m_{\tilde{t}}^{2}}\right], \\
C_{H W} & =\frac{g^{2}}{16} \frac{h_{t}^{2}}{(4 \pi)^{2}}\left[\left(1-\frac{1}{6} \frac{c_{2 \beta} g^{\prime 2}}{h_{t}^{2}}\right)-\frac{2}{5} \frac{X_{t}^{2}}{m_{\tilde{t}}^{2}}\right], \\
C_{H W B} & =-\frac{g g^{\prime}}{24} \frac{h_{t}^{2}}{(4 \pi)^{2}}\left[\left(1+\frac{1}{2} \frac{c_{2 \beta} g^{2}}{h_{t}^{2}}\right)-\frac{4}{5} \frac{X_{t}^{2}}{m_{\tilde{t}}^{2}}\right],
\end{aligned}
$$

where $h_{t}$ is the top quark Yukawa coupling, the supersymmetric partners of the left- and right-handed quarks are assumed to have the same mass $m_{\tilde{t}}, X_{t}$ is the off-diagonal term in their mass matrix, and $c_{2 \beta} \equiv \cos 2 \beta$, where $\tan \beta$ is the ratio of supersymmetric Higgs vacuum expectation values. 


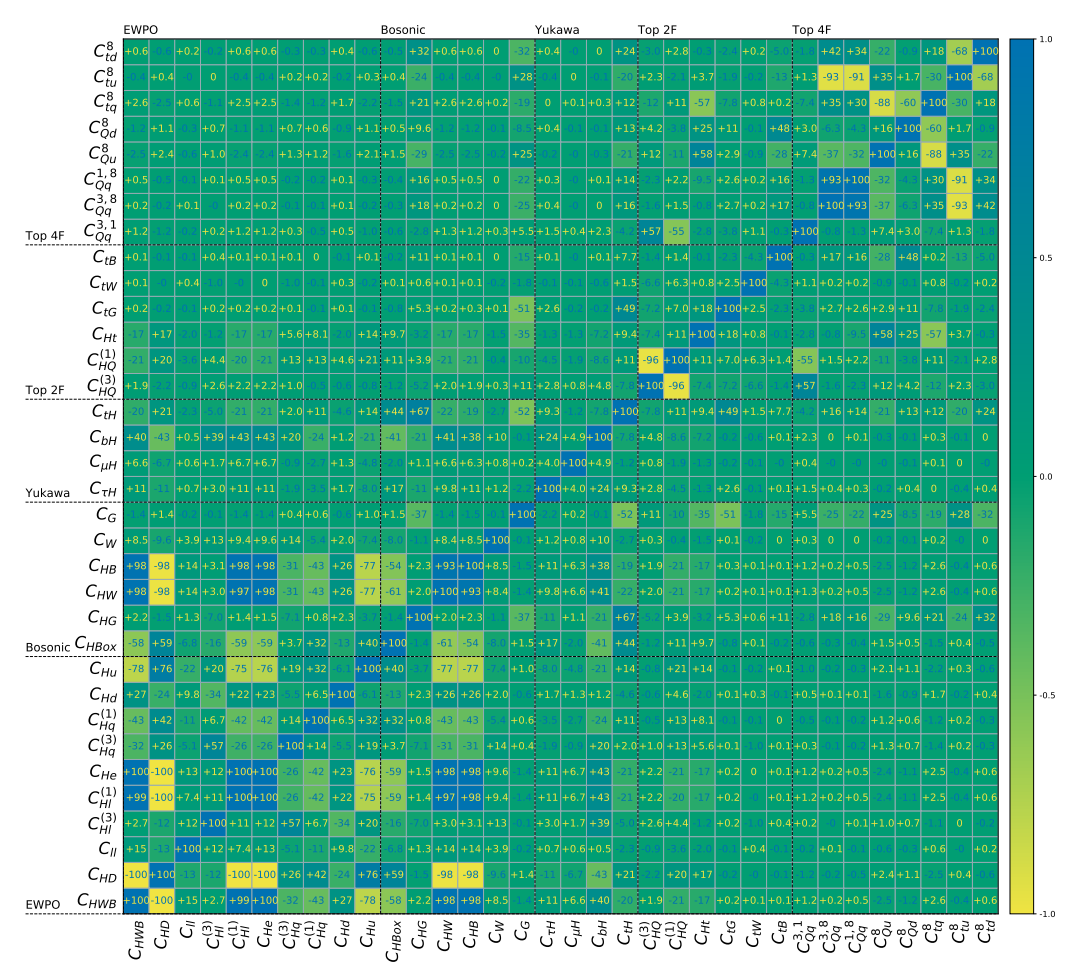

!

FIGURE 4: The correlation matrix for the 34 operator coefficients in the marginalized top-specific fit [16]. The entries are colour-coded according to the magnitudes of the correlation coefficients.

\begin{tabular}{|c|c|c|c|c|c|c|c|c|c|c|c|}
\hline Name & Spin & $\mathrm{SU}(3)$ & $\mathrm{SU}(2)$ & $\mathrm{U}(1)$ & Param. & Name & Spin & SU(3) & SU(2) & U(1) & Param. \\
\hline \hline$S$ & 0 & 1 & 1 & 0 & $\left(M_{S}, \kappa_{S}\right)$ & $\Delta_{1}$ & $\frac{1}{2}$ & 1 & 2 & $-\frac{1}{2}$ & $\left(M_{\Delta_{1}}, \lambda_{\Delta_{1}}\right)$ \\
\hline$S_{1}$ & 0 & 1 & 1 & 1 & $\left(M_{S_{1}}, y_{S_{1}}\right)$ & $\Delta_{3}$ & $\frac{1}{2}$ & 1 & 2 & $-\frac{1}{2}$ & $\left(M_{\Delta_{3}}, \lambda_{\Delta_{3}}\right)$ \\
\hline$\varphi$ & 0 & 1 & 2 & $\frac{1}{2}$ & $\left(M_{\varphi_{1}}, Z_{6} \cos \beta\right)$ & $\Sigma$ & $\frac{1}{2}$ & 1 & 3 & 0 & $\left(M_{\Sigma}, \lambda_{\Sigma}\right)$ \\
\hline$\Xi$ & 0 & 1 & 3 & 0 & $\left(M_{\Xi}, \kappa_{\Xi}\right)$ & $\Sigma_{1}$ & $\frac{1}{2}$ & 1 & 3 & -1 & $\left(M_{\Sigma_{1}}, \lambda_{\Sigma_{1}}\right)$ \\
\hline$\Xi_{1}$ & 0 & 1 & 3 & 1 & $\left(M_{\Xi_{1}}, \kappa_{\Xi_{1}}\right)$ & $U$ & $\frac{1}{2}$ & 3 & 1 & $\frac{2}{3}$ & $\left(M_{U}, \lambda_{U}\right)$ \\
\hline$B$ & 1 & 1 & 1 & 0 & $\left(M_{B}, \hat{g}_{H}^{B}\right)$ & $D$ & $\left.\frac{1}{2}\right)$ & 3 & 1 & $-\frac{1}{3}$ & $\left(M_{D}, \lambda_{D}\right)$ \\
\hline$B_{1}$ & 1 & 1 & 1 & 1 & $\left(M_{B_{1}}, g_{B_{1}}\right)$ & $Q_{1}$ & $\frac{1}{2}$ & 3 & 2 & $\frac{1}{6}$ & $\left(M_{Q_{1}}, \lambda_{Q_{1}}\right)$ \\
\hline$W$ & 1 & 1 & 3 & 0 & $\left(M_{W_{1}}, \hat{g}_{H}^{W}\right)$ & $Q_{5}$ & $\frac{1}{2}$ & 3 & 2 & $-\frac{5}{6}$ & $\left(M_{Q_{5}}, \lambda_{Q_{5}}\right)$ \\
\hline$W_{1}$ & 1 & 1 & 3 & 1 & $\left(M_{W_{1}}, \hat{g}_{W_{1}}^{\varphi}\right)$ & $Q_{7}$ & $\frac{1}{2}$ & 3 & 2 & $\frac{7}{6}$ & $\left(M_{Q_{7}}, \lambda_{Q_{7}}\right)$ \\
\hline$N$ & $\frac{1}{2}$ & 1 & 1 & 0 & $\left(M_{N}, \lambda_{N}\right)$ & $T_{1}$ & $\frac{1}{2}$ & 3 & 3 & $-\frac{1}{3}$ & $\left(M_{T_{1}}, \lambda_{T_{1}}\right)$ \\
\hline$E$ & $\frac{1}{2}$ & 1 & 1 & -1 & $\left(M_{E}, \lambda_{E}\right)$ & $T_{2}$ & $\frac{1}{2}$ & 3 & 3 & $\frac{2}{3}$ & $\left(M_{T_{2}}, \lambda_{T_{2}}\right)$ \\
\hline$T$ & $\frac{1}{2}$ & 3 & 1 & $\frac{2}{3}$ & $\left(M_{T}, S_{L}^{t}\right)$ & $T B$ & $\frac{1}{2}$ & 3 & 2 & $\frac{1}{6}$ & $\left(M_{T B}, S_{L}^{t, b}\right)$ \\
\hline
\end{tabular}

Single-field extensions of the SM analyzed in [16].

Fig. 8 shows the constraint in the $\left(X_{t}, m_{\tilde{t}}\right)$ plane for $\tan \beta=20$ that is imposed by our SMEFT analysis. We see that values of $m_{\tilde{t}} \gtrsim 300 \mathrm{GeV}$ are favoured for most values of $X_{t}$. It is interesting to compare this indirect constraint with constraints from direct searches for stop squarks at the LHC. These are quite complex, since the production and decays of stop squarks depend on several othe supersymmetric model parameters, and some decay modes can be difficult to distinguish from Standard Model backgrounds. On the other hand, the indirect search using the SMEFT is relatively model-independent and of comparable strength. 


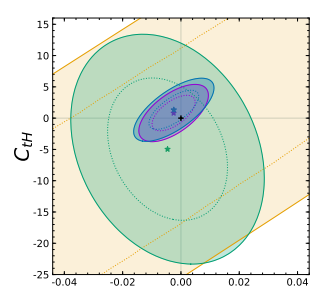

Marginalised 95\% C. L.

Higgs data (not $\bar{t} H$ )

Higgs data

Higgs \& Top data

Higgs \& Top data (+4F)

SM
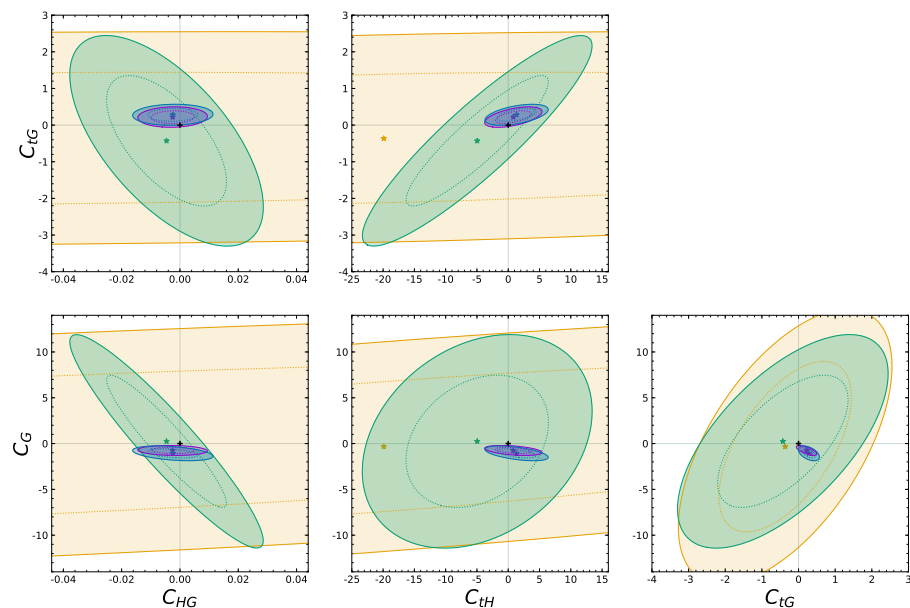

FIGURE 5: Constraints on pairs of operator coefficients at the 95\% confidence level, marginalized over the other degrees of freedom in the 'Higgs only' operator set, obtained using the sets of data indicated by colour coding [16].

\section{MODEL-INDEPENDENT BSM SURVEY}

In addition to these searches for specific extensions of the Standard Model, we have also made a broad-band search for generic models that contribute to arbitrary combinations of 2, 3, 4 or 5 operator coefficients. For all of these 61, 5984, 46736 and 278256 combinations, we were able to calculate the corresponding improvement in the global fit they provide in the forms of their pulls. The resulting pull distributions are shown in Fig. 9 as histograms for combinations containing only $t \bar{t}$ operators (blue), no $t \bar{t}$ operators (yellow) and both types of operator (green). The expected 95\% ranges for the pull distributions are shown as dashed vertical lines. As expected, some pulls lie outside these range, particularly those containing $t \bar{t}$ operators. However, these tails of the distributions are not excessively large, and we again see no significant evidence for new physics beyond the Standard Model.

\section{CONSTRAINING DIMENSION-8 TERMS IN THE SMEFT}

What about the contributions of SMEFT operators with dimension 8 ? These are difficult to isolate, as their contributions are usually smothered by those of dimension 6. However, there are three interesting processes where the leading SMEFT operators have dimension 8 , providing novel windows on possible new physics beyond the Standard Model.

The first of these is light-by-light scattering, $\gamma \gamma \rightarrow \gamma \gamma$, which was first observed in peripheral heavy-ion collisions at the LHC [22]. This process can arise from loop diagrams in the Standard Model, as was first calculated by Heisenberg and Euler in the 1930s [23]. Almost simultaneously, Born and Infeld [24] suggested that there might be nonlinearities in QED capable of generating light-by-light scattering, which they motivated by the idea that there should be a maximum electromagnetic field, just as there is a maximum velocity, that of light. Some 50 years later it was shown that such nonlinearities arise in string theory [25], and then it was shown in the 1990s that in brane models they are due to brane velocities being bounded by that of light [26] - a remarkable vindication of the insight of Born and Infeld! In the SMEFT language, the leading nonlinearity in the Born-Infeld Lagrangian is a term of dimension 8. The LHC measurements were consistent with loop calculations in the Standard Model, and could be used to constrain the mass parameter $M=\sqrt{\beta}$ appearing in Born-Infeld theory to be $\gtrsim 200 \mathrm{GeV}$, as seen in Fig. 10 [27], which is the first significant experimental constraint on this parameter.

A second probe of dimension- 8 terms in the SMEFT was provided by the process $g g \rightarrow \gamma \gamma$, which could arise from Born-Infeld terms in the Standard Model [28]. The appearance of such terms has been constrained by measurements of $\gamma \gamma$ final states in $p p$ collisions at the LHC [29]. As seen in Fig. 11, there are 8 dimension- 8 operators that could contribute to this process. The first analysis using early LHC Run 2 data shown in Fig. 11 provided lower limits $\gtrsim 1000 \mathrm{GeV}$ on the scale parameters of all of these operators, and the sensitivity could be improved to $\gtrsim 2 \mathrm{TeV}$ with data from HL-LHC and $\gtrsim 7 \mathrm{TeV}$ at FCC-hh [28]. 


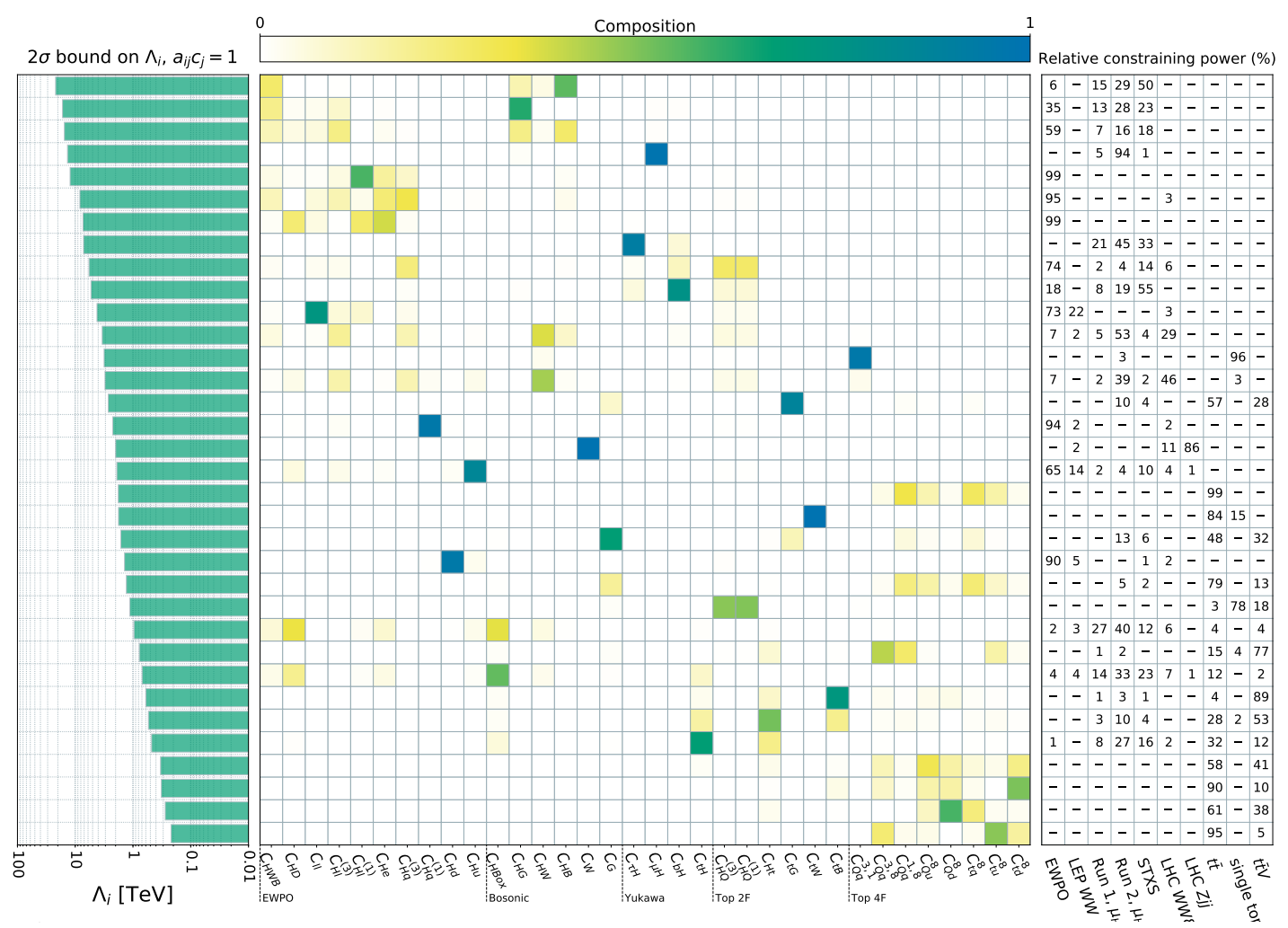

FIGURE 6: Constraints on the eigenvectors of the global likelihood function in a principal component analysis [16]. The left panel shows the strengths of the global constraints on the eigenvectors, the compositions of the eigenvectors are colour-coded in the central panel, and the right panel shows the relative constraining powers of different data sets for each eigenvector. Cases with no significant constraining power are indicated by "-".

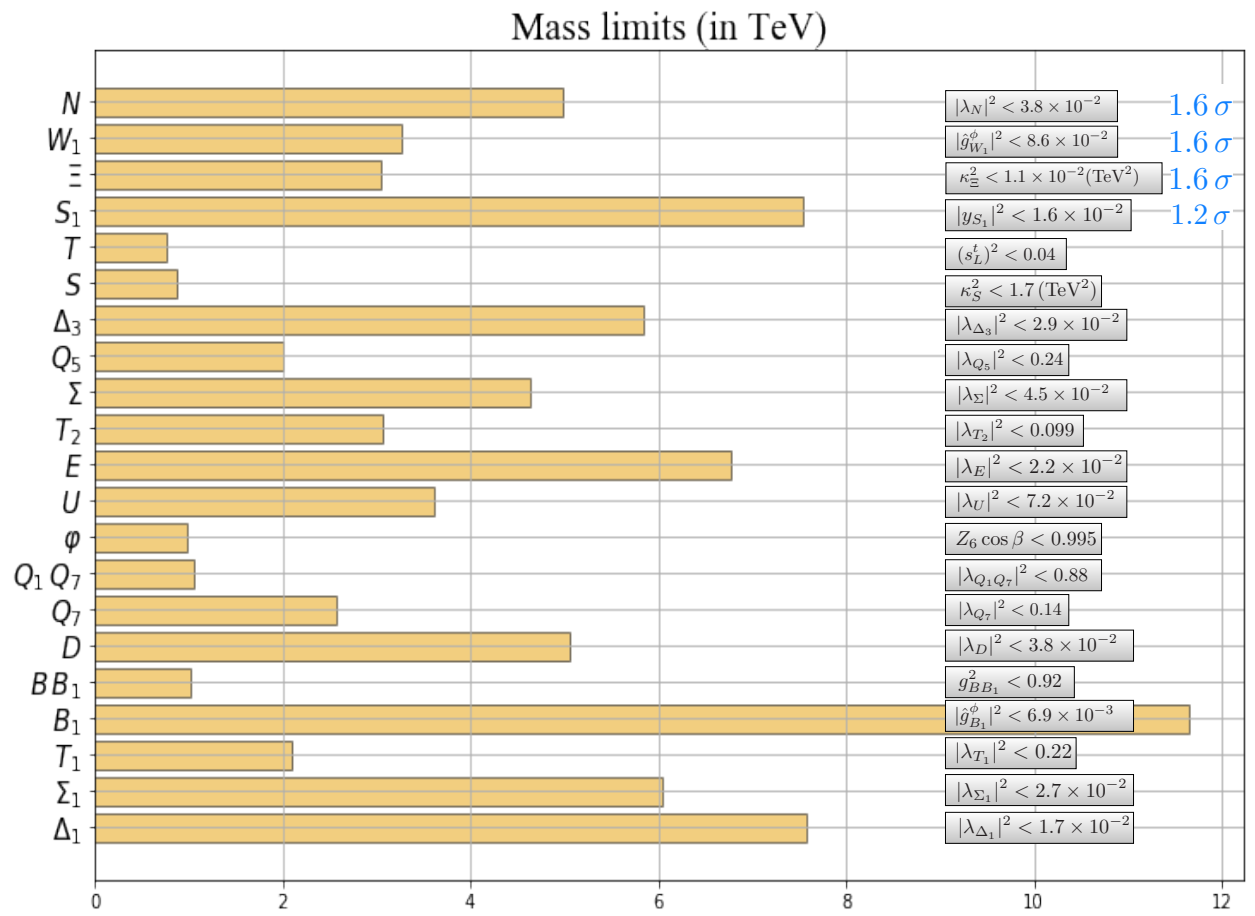

FIGURE 7: Mass limits (in TeV) at the 95\% CL for single-field models assuming that the corresponding couplings are unity, and limits on their couplings assuming masses of $1 \mathrm{TeV}$ [16]. 


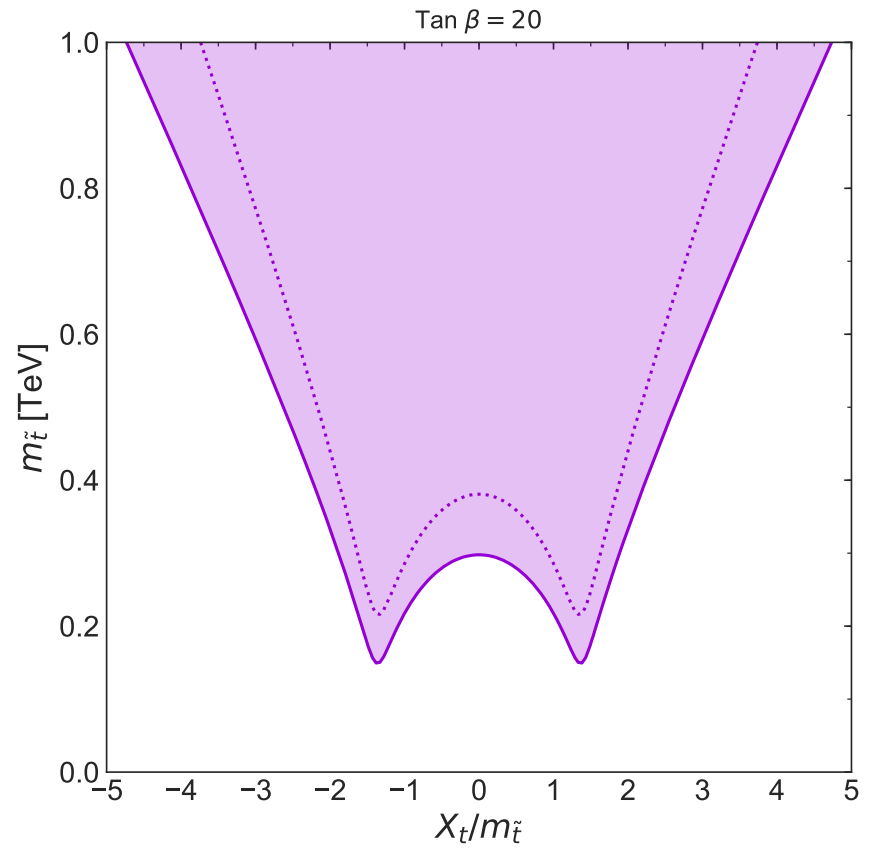

FIGURE 8: SMEFT constraints on the stop parameters $\left(\frac{X_{t}}{m_{\tilde{t}}}, m_{\tilde{t}}\right)$ for $\tan \beta=20$ [16].
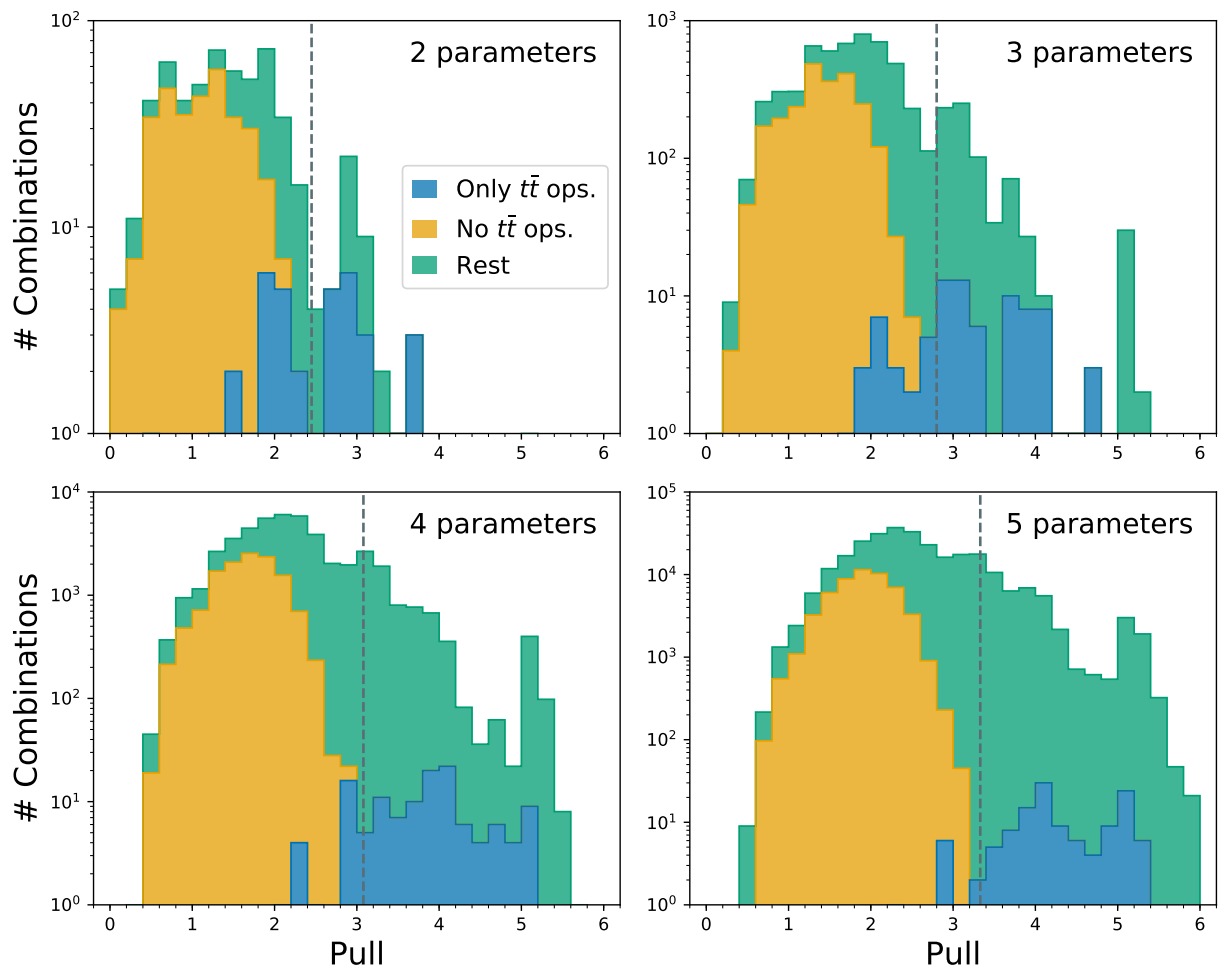

FIGURE 9: Stacked histograms of the pull distributions obtained in fits including 2 (upper left), 3 (upper right), 4 (lower left) and 5 (lower right) dimension-6 SMEFT operators, divided into three categories: those including only operators affecting t $\bar{t}$ production (blue), those that do not affect $t \bar{t}$ production (orange), and the rest (green) [16]. The dashed vertical lines mark the expected 95\% ranges for the pull distributions.

Another opportunity to probe dimension-8 operators at future accelerators is provided by measurements of neutral triplegauge-boson vertices (nTGVs), e.g., via the process $e^{+} e^{-} \rightarrow Z_{\gamma}[30,31]$. The $Z_{\gamma}$ and $Z_{\gamma \gamma}$ nTGVs are absent in the Standard Model Lagrangian, and are not generated by dimension-6 SMEFT terms, either. However, several types of nTGVs are generated by dimension-8 SMEFT operators, and generate $Z_{\gamma}$ final states in $e+e^{-}$collisions with distinctive angular distributions that enable 


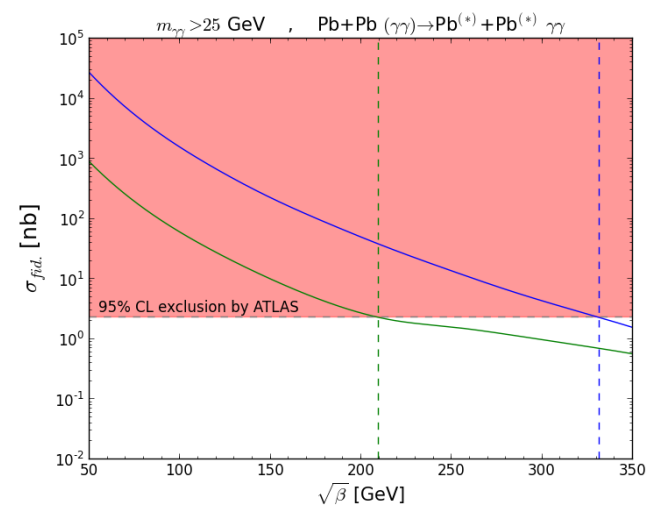

FIGURE 10: The fiducial cross section for light-by-light scattering in relativistic heavy-ion collisions, $\sigma\left(\mathrm{Pb}+\mathrm{Pb}(\gamma \gamma) \rightarrow \mathrm{Pb}^{(*)}+\mathrm{Pb}^{(*)} \gamma \gamma\right)$ as a function of $M=\sqrt{\beta}$ in Born-Infeld theory obtained with different theoretical assumptions (green and blue lines) is compared with the 95\% CL ATLAS upper limit [22], which excludes the range shaded pink [27].
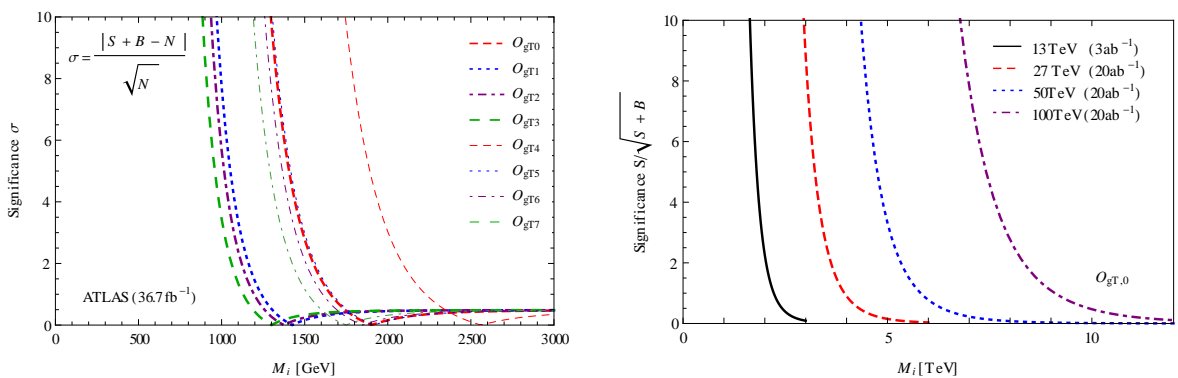

FIGURE 11: Sensitivities to the new physics scales $\Lambda$ of the indicated dimension-8 operators contributing to gg $\rightarrow \gamma \gamma$ in (a) early LHC Run 2 data and (b) possible future data at the LHC and proposed pp colliders [28].

them to be distinguished from Standard Model backgrounds. Fig. 12 summarizes the potential 2- and 5- $\sigma$ sensitivities to the scales of dimension- 8 operators provided by $2 \mathrm{ab}^{-1}$ of integrated luminosity in unpolarized (left panel) and polarized (right panel) $e^{+} e^{-}$ collisions at a range of centre-of-mass energies from $250 \mathrm{GeV}$ (corresponding to FCC-ee, CEPC or the initial stage of the ILC) to $3 \mathrm{TeV}$ (the target of CLIC) and above [31]. The possible sensitivities reach into the multi-TeV range, depending on the operator considered, and improve if the $e^{ \pm}$beams are polarized.
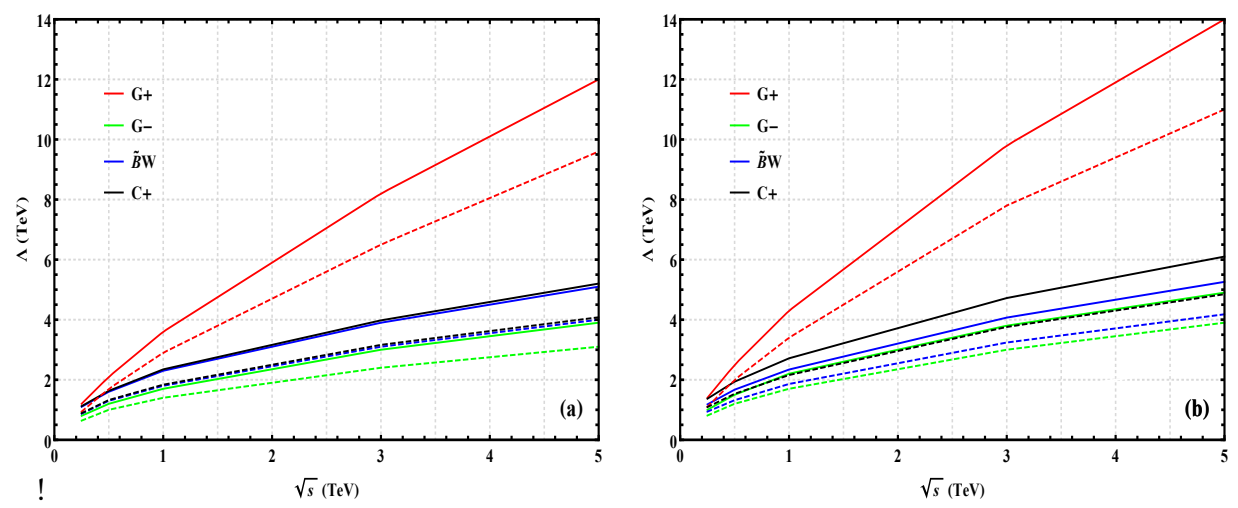

FIGURE 12: Sensitivities to the new physics scales $\Lambda$ of the indicated dimension-8 operators contributing to $n T G V s$, as functions of the $e^{+} e^{-}$ collision energy $\sqrt{s}$ at the 2- and 5- $\sigma$ levels (solid and dashed lines), assuming an integrated luminosity of 2 ab ${ }^{-1}$ and (a) unpolarized and (b) polarized beams [31].

\section{SUMMARY}

Effective field theories have served particle physics well in the past, indicating the direction to follow in constructing the electroweak sector of the Standard Model, and also the structure of the gluon interactions underlying the strong force. Many extensions of the Standard Model predict new particles and/or interactions at scales beyond the masses of the known particles, which 


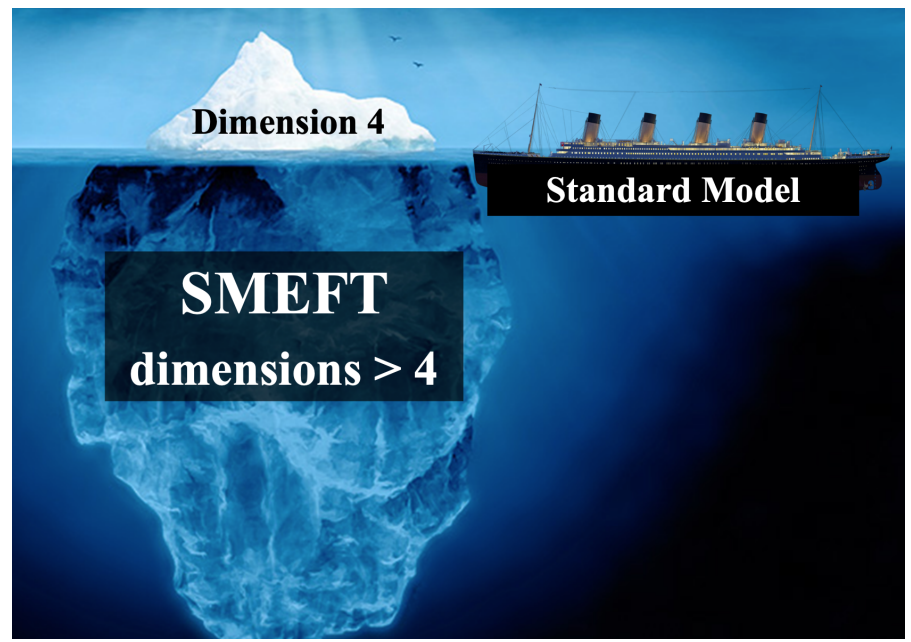

FIGURE 13: Will the Standard Model soon be sunk by SMEFT interactions with dimensions > 4?

may show up first as additional interactions in the Standard Model Effective Field Theory (SMEFT) that indicate the direction in which new physics may lie. The most prominent among these new interactions are likely to be those of dimension 6 , but there are also opportunities to search directly for possible new interactions of dimension 8 . This talk has summarized key features of a first global analysis of data from Run 2 of the LHC and previous experiments incorporating dimension-6 operators [16], and how its results can be used to constrain possible extensions of the Standard Model. In addition, some of the opportunities for studying dimension-8 SMEFT operators have also been mentioned.

So far, these SMEFT analyses have not provided any significant indication of possible physics beyond the Standard Model. However, one may still hope that the far greater integrated luminosity to be provided by future runs of the LHC will reveal significant deviations from the Standard Model, and indicate how Nature has chosen to go beyond it. The prospects for the future are summarized in Fig. 13. The dimension-4 Lagrangian of the Standard Model is only an infinitesimal part of the full set of possible interactions between its particles that are represented by the SMEFT interactions with dimensions $>4$. So far, the Standard Model has been cruising along untroubled, but it may soon collide with the SMEFT and sink, to be replaced by a more complete theory.

\section{ACKNOWLEDGEMENTS}

It is a pleasure to acknowledge my collaborators on the work described here: Ola Drozd, Shaofeng Ge, Hongjian He, Maeve Madigan, Nick Mavromatos, Ken Mimasu, Chris Murphy, Jérémie Quevillon, Veronica Sanz, Ruiqing Xiao and Tevong You. The work described here was supported by the United Kingdom STFC via grant ST/T000759/1, and by the Estonian Research Council via a Mobilitas Pluss grant.

\section{References}

[1] ALEPH, CDF, D0, DELPHI, L3, OPAL and SLD Collaborations, LEP Electroweak Working Group, Tevatron Electroweak Working Group, SLD Electroweak and Heavy Flavour Groups, "Precision Electroweak Measurements and Constraints on the Standard Model", arXiv:1012.2367 [hep-ex].

[2] G. Aad et al. [ATLAS Collaboration], Phys. Lett. B 716 (2012), 1-29 doi:10.1016/j.physletb.2012.08.020 [arXiv:1207.7214 [hep-ex]]; S. Chatrchyan et al. [CMS Collaboration], Phys. Lett. B 716 (2012), 30-61 doi:10.1016/j.physletb.2012.08.021 [arXiv:1207.7235 [hep-ex]].

[3] CMS Collaboration, https://twiki.cern.ch/twiki/bin/view/CMSPublic/PhysicsResultsCombined\#EXO_summary_plot.

[4] ATLAS Collaboration, https://atlas.web.cern.ch/Atlas/GROUPS/PHYSICS/CombinedSummaryPlots/HIGGS/.

[5] J. Ellis and T. You, JHEP 09 (2012), 123 doi:10.1007/JHEP09(2012)123 [arXiv:1207.1693 [hep-ph]].

[6] D. Buttazzo, G. Degrassi, P. P. Giardino, G. F. Giudice, F. Sala, A. Salvio and A. Strumia, JHEP 12 (2013), 089 doi:10.1007/JHEP12(2013)089 [arXiv:1307.3536 [hep-ph]].

[7] A. Hook, J. Kearney, B. Shakya and K. M. Zurek, JHEP 01 (2015), 061 doi:10.1007/JHEP01(2015)061 [arXiv:1404.5953 [hep-ph]].

[8] Sun Tzu, The Art of War, translated by Samuel B. Griffith (Oxford University Press, 1963) ISBN 978-0-19-501476-1. I am grateful to Ken Mimasu for this quotation.

[9] E. Fermi, Z. Phys. 88 (1934), 161-177 doi:10.1007/BF01351864.

[10] R. P. Feynman and M. Gell-Mann, Phys. Rev. 109 (1958), 193-198 doi:10.1103/PhysRev.109.193.

[11] F. Englert and R. Brout, Phys. Rev. Lett. 13 (1964), 321-323 doi:10.1103/PhysRevLett.13.321; P. W. Higgs, Phys. Lett. 12 (1964), 132-133 doi:10.1016/0031-9163(64)91136-9 and Phys. Rev. Lett. 13 (1964), 508-509 doi:10.1103/PhysRevLett.13.508

[12] S. Weinberg, Phys. Rev. Lett. 43 (1979), 1566-1570 doi:10.1103/PhysRevLett.43.1566

[13] W. Buchmuller and D. Wyler, Nucl. Phys. B 268 (1986), 621-653 doi:10.1016/0550-3213(86)90262-2

[14] B. Grzadkowski, M. Iskrzynski, M. Misiak and J. Rosiek, JHEP 10 (2010), 085 doi:10.1007/JHEP10(2010)085 [arXiv:1008.4884 [hep-ph]].

[15] J. Ellis, C. W. Murphy, V. Sanz and T. You, JHEP 06 (2018), 146 doi:10.1007/JHEP06(2018)146 [arXiv:1803.03252 [hep-ph]].

[16] J. Ellis, M. Madigan, K. Mimasu, V. Sanz and T. You, JHEP 04 (2021), 279 doi:10.1007/JHEP04(2021)279 [arXiv:2012.02779 [hep-ph]].

[17] C. Hays, V. Sanz Gonzalez and G. Zemaityte, "Constraining EFT parameters using simplified template cross sections", LHCHXSWG-2019-004. 
[18] J. J. Ethier, F. Maltoni, L. Mantani, E. R. Nocera, J. Rojo, E. Slade, E. Vryonidou and C. Zhang, arXiv:2105.00006 [hep-ph].

[19] J. de Blas, J. C. Criado, M. Perez-Victoria and J. Santiago, JHEP 03 (2018), 109 doi:10.1007/JHEP03(2018)109 [arXiv:1711.10391 [hep-ph]].

[20] A. Drozd, J. Ellis, J. Quevillon and T. You, JHEP 06 (2015), 028 doi:10.1007/JHEP06(2015)028 [arXiv:1504.02409 [hep-ph]].

[21] B. Henning, X. Lu and H. Murayama, "What do precision Higgs measurements buy us?", arXiv:1404.1058 [hep-ph].

[22] M. Aaboud et al. [ATLAS Collaboration], Nature Phys. 13 (2017) no.9, 852-858 doi:10.1038/nphys4208 [arXiv:1702.01625 [hep-ex]].

[23] W. Heisenberg and H. Euler, Z. Phys. 98 (1936) no.11-12, 714-732 doi:10.1007/BF01343663 [arXiv:physics/0605038 [physics]].

[24] M. Born and L. Infeld, Proc. Roy. Soc. Lond. A 144 (1934) no.852, 425-451 doi:10.1098/rspa.1934.0059

[25] E. S. Fradkin and A. A. Tseytlin, Phys. Lett. B 163 (1985), 123-130 doi:10.1016/0370-2693(85)90205-9

[26] C. Bachas, Phys. Lett. B 374 (1996), 37-42 doi:10.1016/0370-2693(96)00238-9 [arXiv:hep-th/9511043 [hep-th]].

[27] J. Ellis, N. E. Mavromatos and T. You, Phys. Rev. Lett. 118 (2017) no.26, 261802 doi:10.1103/PhysRevLett.118.261802 [arXiv:1703.08450 [hepph]].

[28] J. Ellis and S. F. Ge, Phys. Rev. Lett. 121 (2018) no.4, 041801 doi:10.1103/PhysRevLett.121.041801 [arXiv:1802.02416 [hep-ph]].

[29] M. Aaboud et al. [ATLAS Collaboration], Phys. Lett. B 775 (2017), 105-125 doi:10.1016/j.physletb.2017.10.039 [arXiv:1707.04147 [hep-ex]].

[30] J. Ellis, S. F. Ge, H. J. He and R. Q. Xiao, Chin. Phys. C 44 (2020) no.6, 063106 doi:10.1088/1674-1137/44/6/063106 [arXiv:1902.06631 [hep-ph]].

[31] J. Ellis, H. J. He and R. Q. Xiao, Sci. China Phys. Mech. Astron. 64 (2021) no.2, 221062 doi:10.1007/s11433-020-1617-3 [arXiv:2008.04298 [hep$\mathrm{ph}]$. 